\title{
Improving water management efficiency by using optimization-based control strategies: the Barcelona case study
}

\author{
C. Ocampo-Martinez, V. Puig, G. Cembrano, R. Creus and M. Minoves
}

\begin{abstract}
$\overline{\text { ABSTRACT }}$
This paper describes the application of model-based predictive control (MPC) techniques to the flow management in large-scale drinking water networks including a telemetry/telecontrol system. MPC technique is used to generate flow control strategies from the sources to the consumer areas to meet future demands, optimizing performance indexes associated to operational goals such as economic cost, network safety volumes and flow control stability. The designed management strategies are applied to a real case study based on a representative model of the drinking water network of Barcelona (Spain).

Key words | drinking water systems, large-scale systems, optimization-based control, predictive control

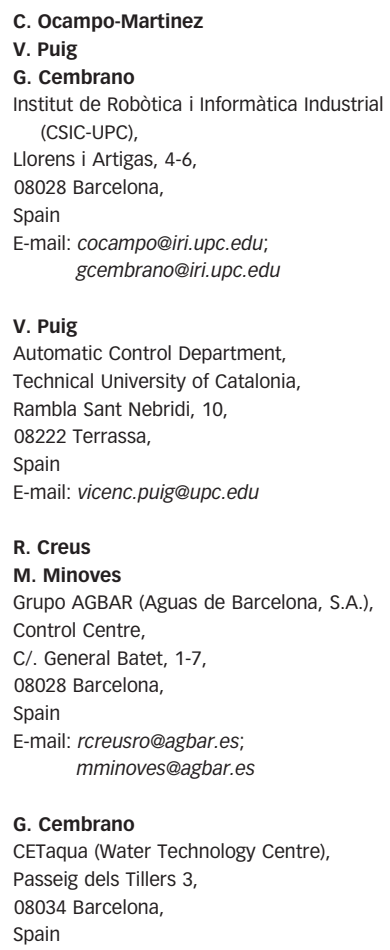

\section{INTRODUCTION}

Drinking water management in urban areas is a subject of increasing concern as cities grow. Limited water supplies, conservation and sustainability policies, as well as the infrastructure complexity for meeting consumer demands with appropriate flow, pressure and quality levels make water management a challenging control problem. Decision support systems provide useful guidance for operators in complex networks, where resources management best actions are not intuitive. Optimization and optimal control techniques provide an important contribution to a smart management strategy computation for drinking water networks (DWN) (see Nitivattananon et al. 1996; Westphal et al. 2003; Tu et al. 2005). Similarly, problems related to modelling and control of water supply, transport and distribution systems have been object of important research efforts during the last few years (see, e.g. Brdys \& Ulanicki 1994; Cembrano et al. 2000; Maksimovic et al. 2003; Butler \& Memon 2006).

This paper describes preliminary results of a collaborative project between AGBAR, the company in charge of water transport and distribution in Barcelona and its metropolitan area and the Advanced Control Systems 
research group (SAC) from the Technical University of Catalonia (UPC). The objective of the project is to apply model-based predictive control techniques for flow management in large-scale water transport systems.

Model predictive control (MPC) is a set of control methodologies that use a mathematical model of the considered system to obtain control signals over a time horizon that minimize a cost function related to selected indexes associated to a desired system performance. MPC is very flexible regarding its implementation and can be used over almost all systems since it is set according to the model of the plant (Maciejowski 2002; Camacho \& Bordons 2004). Moreover, MPC has some features to deal with complex systems, such as water networks, which present the following characteristics: physical and operational constraints, a multivariable and large-scale nature, demand forecasting requirement, and complex, multi-objective operational goals. Moreover, MPC is relatively simple to be used by people without deep knowledge of control. Thus, according to (Marinaki \& Papageorgiou 2005; Ocampo-Martinez 2007; Brdys et al. 2008), among others, such controllers are very suitable to be used in the global control of networks related to the urban water cycle within a hierarchical control structure. This global control structure is shown in Figure 1, where the MPC determines the references for the local controllers located on different

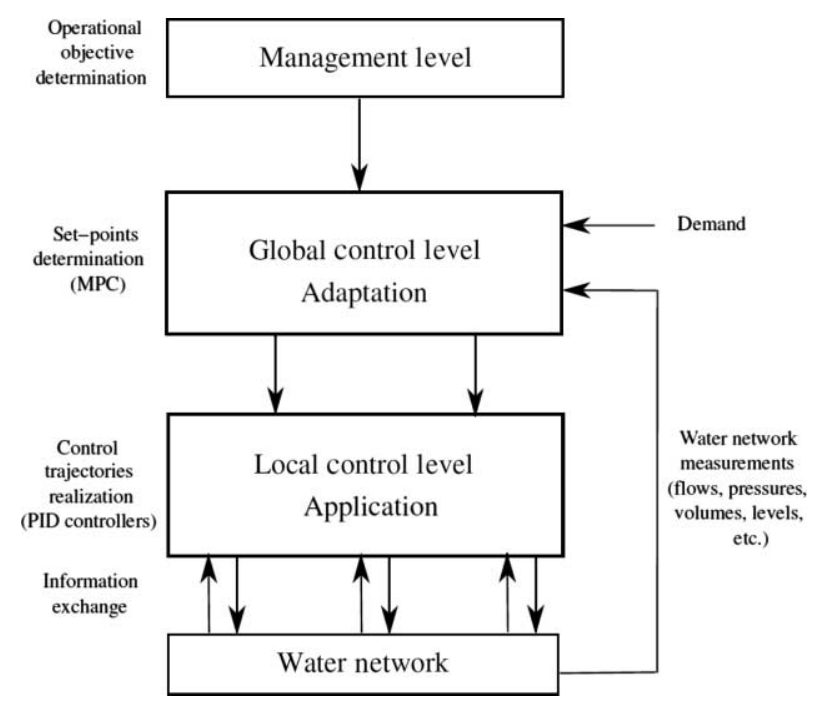

Figure 1 | Hierarchical structure for RTC of drinking water system. Adapted from (Ocampo-Martinez 2007). elements of the network. The management level is used to provide MPC with the operational objective, which is reflected in the controller design as the performance indexes to be optimized.

In general, DWNs are pressurized systems containing multiple tanks, pumping stations, water sources (superficial and underground) and sectors of consumer demand (Brdys \& Ulanicki 1994). The MPC technique is used here to generate flow-control strategies from the drinking water treatment plants to the consumer areas to meet future demands, optimizing a performance index expressing operational goals such as economic cost, water safety storage and flow control stability. The main contribution of this paper consists in highlighting the advantages of using optimization-based control techniques as MPC to improve the performance of a DWN taking into account the added complexity of the MPC design for these systems, namely, their large scale (in terms of number of dynamic elements and decision variables), the nature of the desired control objectives and the type and behaviour of the system disturbances (drinking water demands). The developed control strategies have been tested on the drinking water transport network of Barcelona, a representative example of a large-scale and complex DWN.

The structure of the paper is as follows: first, modelling and MPC applied to DWNs is revised. Then, the description of the case study based on an aggregate model of the Barcelona drinking water system is presented. Next, the main results obtained from simulations of the closed-loop system using MPC techniques are discussed. Different aspects and issues of the simulations are commented on. Finally, the main conclusions are drawn.

\section{DWN MODELLING ORIENTED TO PREDICTIVE CONTROL}

In order to obtain the DWN control-oriented model, the constitutive elements and basic relationships are introduced.

The mass balance expression relating the stored volume in tanks, $x$, the manipulated tank inflows and outflows, $u$, and the demands, $d$, can be written as 
the difference equation

$x_{i}(k+1)=x_{i}(k)+\Delta t\left(\sum_{i} q_{\mathrm{in}, i}(k)-\sum_{j} q_{\mathrm{out}, j}(k)\right)$

where $q_{\mathrm{in}, i}(k)$ and $q_{\mathrm{out}, j}(k)$ correspond to the $i$-th tank inflow and the $j$-th tank outflow, respectively, given in $\mathrm{m}^{3} / \mathrm{s}$. The physical constraint related to the range of tank volume capacities is expressed as

$x^{\min } \leq x(k) \leq x^{\max }$

where $x^{\min }$ and $x^{\text {max }}$ denote the minimum and the maximum volume capacity, respectively, given in $\mathrm{m}^{3}$. Since this is a physical limit, it is expressed as a hard constraint: it is impossible to send more water to a tank than it can store.

In a DWN, nodes correspond to intersections of mains. The static equation that expresses the mass conservation in these elements can be written as

$\sum_{i} q_{\mathrm{in}, i}(k)=\sum_{j} q_{\mathrm{out}, j}(k)$

where $q_{\text {in, } i}(k)$ and $q_{\text {out }, j}(k)$ correspond to the $i$-th node inflow and the $j$-th node outflow, respectively, given in $\mathrm{m}^{3} / \mathrm{s}$. Therefore, considering the expressions presented above, the control-oriented model of a DWN in discretetime state space may be written as:

$x(k+1)=A x(k)+B u(k)+B_{p} d(k)$,

where $x \in \mathbb{R}^{n}$ is the state vector corresponding to the water volumes of the tanks at time $k, u \in \mathbb{R}^{m}$ represents the vector of manipulated flows through the actuators, and $d \in \mathbb{R}^{p}$ corresponds to the vector of demands. $A, B$, and $B_{p}$ are the system matrices of suitable dimensions. Since the demands can be forecasted and they are assumed to be known, $d$ is a known vector containing the measured disturbances affecting the system. Therefore, (4) can be rewritten as

$x(k+1)=A x(k)+\Gamma v(k)$,

where $\Gamma=\left[\begin{array}{ll}B & B_{p}\end{array}\right]$ and $v(k)=\left[\begin{array}{ll}u(k)^{\mathrm{T}} & d(k)^{\mathrm{T}}\end{array}\right]^{\mathrm{T}}$. Regarding the system constraints and according to the network modelling, they are related to:

- Mass balance relationships at the network nodes (relations between manipulated inputs and, in some cases, measured disturbances). These equalities are written as

$$
E_{1} v(k)=E_{2}
$$

- Bounds on system states and measured inputs expressed by (2) and the inequality

$u^{\min } \leq u(k) \leq u^{\max }$

where $u^{\text {min }}$ and $u^{\max }$ are vectors with the lower and upper limits of the actuators, respectively.

Hence, expressions in (2), (5), (6) and (7) constitute the set of constraints related to the DWN mathematical model.

\section{OPTIMIZATION-BASED CONTROL OF DWNS}

Along the last few years, MPC has shown to be one of the most effective and accepted control strategies for complex systems (Maciejowski 2002). The objective of using this technique for controlling DWNs is to compute, in a predictive way, the best manipulated inputs in order to achieve the optimal performance of the network according to a given set of control objectives and predefined performance indexes. MPC strategies have some important features to deal with complex systems, such as water networks: the amenability to including disturbance (demand) prediction, physical constraints and multivariable system dynamics and objectives in a relatively simple fashion.

\section{Control objectives and cost function}

This paper considers that a MPC design for a DWN should satisfy the operational objectives described as follows.

\section{Minimizing water production and transport cost}

The main economic costs associated to drinking water production (treatment) are due to: chemicals, legal canons and electricity costs. Delivering this drinking water to appropriate pressure levels through the water transport network involves important electricity costs in pumping stations. For this study, this control objective can be 
described by the expression

$f_{1}(k)=W_{\alpha}(\alpha u(k))+W_{\gamma}(\gamma(k) u(k))$,

where $\alpha$ corresponds to a known vector related to the economic costs of the water according to the selected source (treatment plant, dwell, etc.) and $\gamma(k)$ is a vector of suitable dimensions associated to the economic cost of the flow through certain actuators (pumps only) and their control cost (pumping). Note the $k$-dependence of $\gamma$ since the pumping effort has different values according to the time of the day (electricity costs). Weight matrices $W_{\alpha}$ and $W_{\gamma}$ penalize the control objective related to economic costs in the optimization process.

\section{Safety storage term}

The satisfaction of water demands should be fulfilled at any time instant. This is guaranteed through the equality constraints of the water mass balances at demand sectors. However, some risk prevention mechanisms should be introduced in the tank management so that, additionally, the stored volume is preferably maintained over safety limit for eventual emergency needs and to guarantee future availability. A quadratic expression for this concept is used, as follows:

$f_{2}(k)=\left\{\begin{array}{ccc}0 & \text { if } & x(k) \geq \beta \\ (x(k)-\beta)^{\mathrm{T}} W_{x}(x(k)-\beta) & \text { if } & x(k)<\beta\end{array}\right.$,

where $\beta$ is a term which determines the security volume to be considered for the control law computation and matrix $W_{x}$ defines the weight of the objective in the cost function.

\section{Stability of control actions}

Pumping stations must, in general, avoid excessive switching; valves should operate smoothly in order to avoid big transients in the pressurized pipes which can lead to poor pipe condition. Similarly water flows requested from treatment plants must have a smooth profile due to the plants operational constraints. To obtain such smoothing effect, the proposed MPC controller includes a third term in the objective function to penalize control signal variation between consecutive time intervals, i.e. $\Delta u(k)=u(k)-u(k-1)$. This term is expressed as

$f_{3}(k)=\Delta u(k)^{\mathrm{T}} W_{u} \Delta u(k)$,

where $\Delta u(k)$ corresponds to the vector of variation in the inputs from time $k-1$ to time $k$, and $W_{u}$ corresponds to a weight matrix of suitable dimensions.

Therefore, the performance function $J(k)$, considering the aforementioned control objectives has the form

$J(k)=\sum_{i=0}^{H_{\mathrm{u}}-1} f_{1}(k+i)+\sum_{i=0}^{H_{\mathrm{p}}} f_{2}(k+i)+\sum_{i=0}^{H_{\mathrm{u}}-1} f_{3}(k+i)$,

where $H_{p}$ and $H_{u}$ correspond to the prediction and control horizons, respectively. In this equation, index $k$ represents the current time instant while index $i$ represents the time along the prediction and control horizons.

The highest priority objective is the economic cost, which should be minimized while obtaining acceptable satisfaction of security and stability objectives. Further improvements in objective priority handling can be obtained by using a lexicographic approach as suggested in (Ocampo-Martínez et al. 2008).

Remark 1. Weight matrices $W_{\alpha}$ and $W_{\gamma}$ for the first control objective, $W_{x}$ for the second, and $W_{x}$ for the third objective are defined as diagonal matrices of the form

$W_{i}=\operatorname{diag}\left[\begin{array}{llll}\sigma_{1} & \sigma_{2} & \ldots & \sigma_{q}\end{array}\right]$

where $i \in\{\alpha, \gamma, x, u\}$ and $\sigma$ is the specific weight for the corresponding variable. Moreover, $q \in\{n, m, p\}$, depending on the variables associated to the cost function term and defining a weight matrix of suitable dimensions.

\section{DEMAND FORECASTING}

The demand forecasting algorithm used for the design procedure of the MPC controller consists of two levels:

- a time-series modelling to represent the daily aggregate flow values, and

- a set of different daily flow demand patterns according to the day type to cater for different consumption during the weekends and holidays periods. Every pattern consists of 24 hourly values for each daily pattern. 
This algorithm runs in parallel with the MPC controller.

The daily series of hourly flow predictions is computed as a product of the daily aggregate flow value and the appropriate hourly demand pattern.

\section{Aggregate daily flow model}

The aggregate daily flow model is built on the basis of a time series modelling approach using ARIMA strategy. A time series analysis was carried out on several daily aggregate series, which consistently showed a weekly seasonality, as well as the presence of deterministic periodic components. A general expression for the aggregate daily flow model, to be used for a number of demands in different locations, was derived using three main components:

- A weekly-period oscillating signal, with zero average value to cater for cyclic deterministic behaviour, implemented using a second-order (two-parameter) model with two oscillating modes in $s$-plane: $s_{1-2}= \pm 2 \pi / 7 j$ (or equivalently, in $z$-plane: $\left.z_{1-2}=\cos (2 \pi / 7) \pm j \sin (2 \pi / 7)\right)$. Then, the oscillating polynomial is:

$$
y(k)=2 \cos \left(\frac{2 \pi}{7}\right) y(k-1)-y(k-2) .
$$

- An integrator takes into account possible trends and the non-zero mean value of the flow data:

$y(k)=y(k-1)$.

- An autoregressive component to consider the influence of previous flow values within a week. For the general case, the influence of four previous days is considered. However, after parameter estimation and significance analysis, the models are usually reduced implementing a smaller number of parameters

$$
\begin{gathered}
y(k)=-a_{1} y(k-1)-a_{2} y(k-2)-a_{3} y(k-3)-a_{4} \\
y(k-4) .
\end{gathered}
$$

Combining the three components (12)-(14) in the following way:

$$
\begin{aligned}
\Delta y_{\mathrm{int}}(k)=y(k)-y(k-1) & \\
\Delta y_{\mathrm{osc}}(k)= & \Delta y_{\mathrm{int}}(k)-2 \cos (2 \pi / 7) \Delta y_{\mathrm{int}}(k-1) \\
& +\Delta y_{\mathrm{int}}(k-2) \\
y_{p}(k)= & -a_{1} \Delta y_{\mathrm{osc}}(k-1)-a_{2} \Delta y_{\mathrm{osc}}(k-2) \\
& -a_{3} \Delta y_{\mathrm{osc}}(k-3)-a_{4} \Delta y_{\mathrm{osc}}(k-4)
\end{aligned}
$$

the structure of aggregate daily flow model for each demand sensor is therefore:

$y_{p}(k)=-b_{1} y(k-1)-b_{2} y(k-2)-\cdots-b_{7} y(k-7)$.

The parameters $b_{1}, \ldots, b_{7}$ should be adjusted using leastsquares-based parameter estimation methods and historical data (after pre-processing to obtain fault-free set). In parallel with the forecasting and control module, a data validation module should be considered, which validates the used information. This part of the research is underway and it is not addressed in this paper. However, the reader is referred to (Ragot \& Maquin 2006) for a methodology that can be used to guarantee that measurements are free of faults.

\section{1-hour flow model}

The 1-hour flow model is based on distributing the daily flow prediction provided by the time-series model described in previous section using a one-hour-flow pattern that takes into account the daily/monthly variation in the following way:

$y_{p 1 h}(k+i)=\frac{y_{\text {pat }}(k, i)}{\sum_{j=1}^{24} y_{\text {pat }}(k, i)} y_{p}(j), \quad i=1, \ldots, 24$

where $y_{p 1 h}(k)$ is the predicted flow for the current day $j$ using (15) and $y_{\text {pat }}(k, i)$ is the prediction provided by the one-hour-flow pattern with the flow pattern class day/ month of the actual day.

\section{$\overline{\text { CASE-STUDY DESCRIPTION }}$}

The water transport network of Barcelona is used as the case study of this paper. This network covers a territorial extension of $425 \mathrm{~km}^{2}$, with a total pipe length of $4,470 \mathrm{~km}$. Every year, it supplies $237.7 \mathrm{hm}^{3}$ of drinking water to a population over 2.8 millions of inhabitants. The network has a centralized telecontrol system, organized in a twolevel architecture. At the upper level, a supervisory control system installed in the control centre of AGBAR is in charge of controlling the whole network by taking into account operational constraints and consumer demands. This upper 


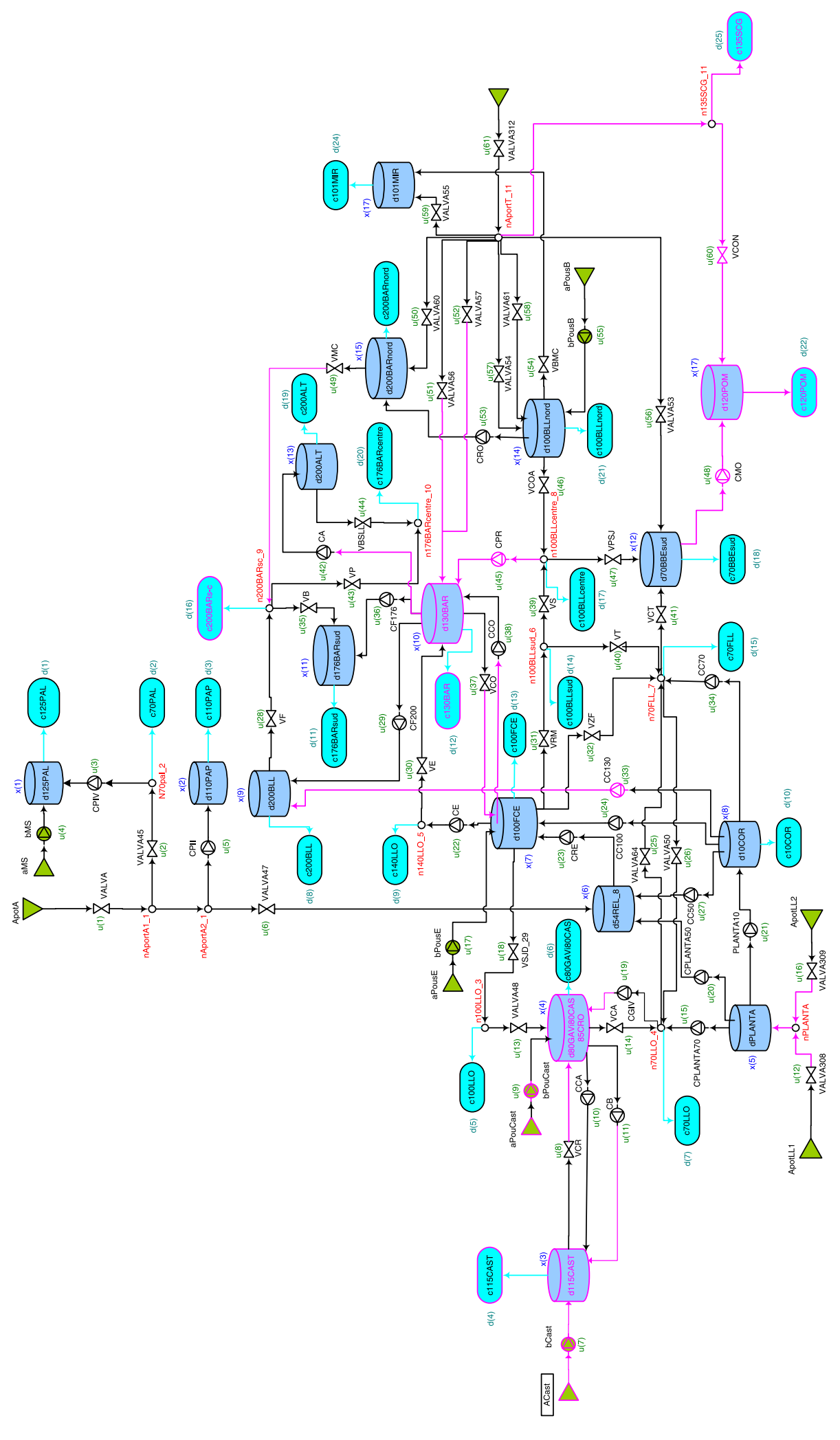

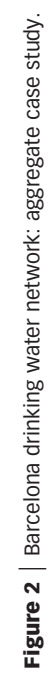




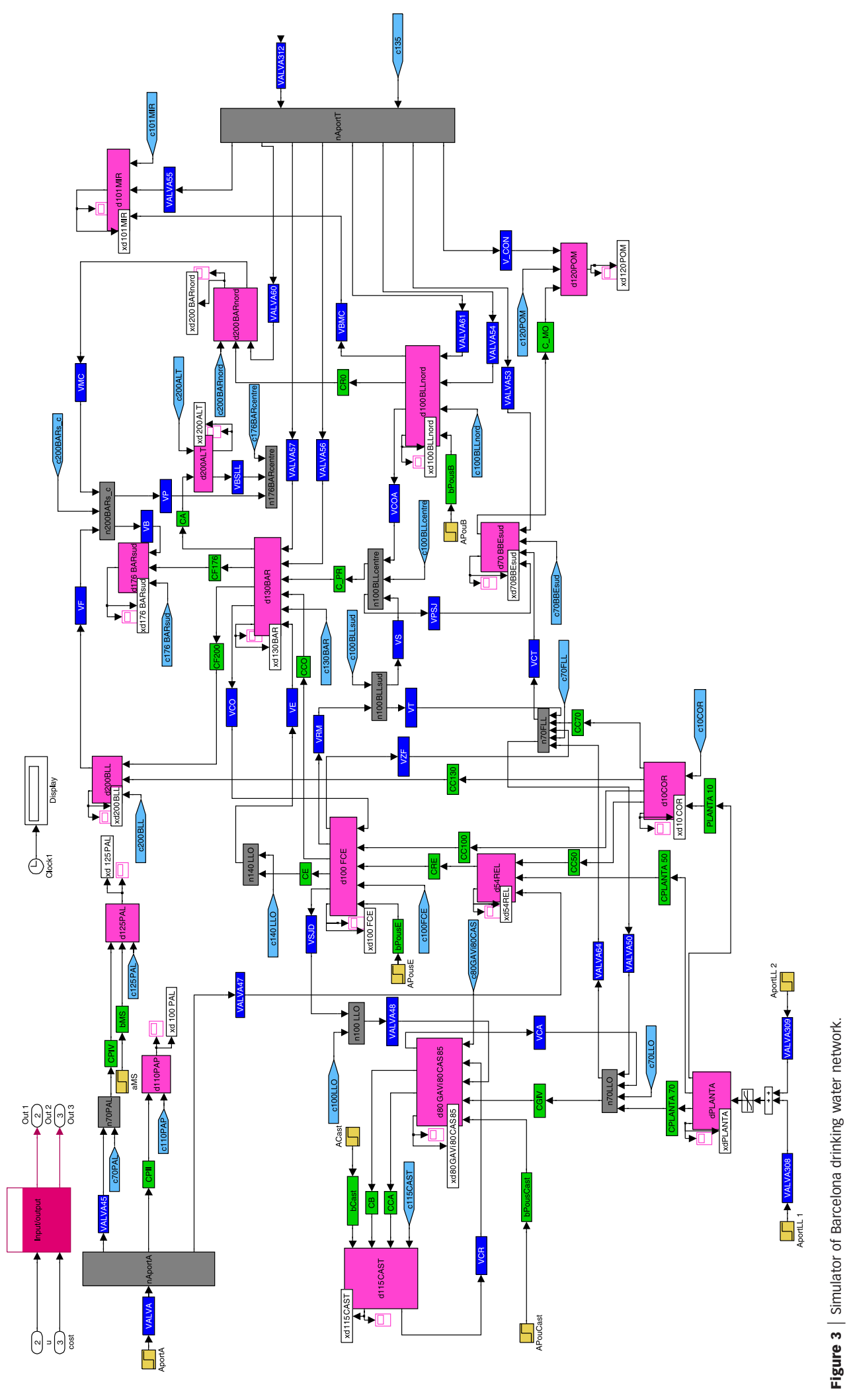


Table 1 | Tuning set-up of the MPC controller

\begin{tabular}{lccc} 
Priority & Economical cost & Water cost & Electricity cost \\
\hline Economical cost & 68.63 & 59.24 & 9.39 \\
Security & 130.93 & 117.84 & 13.09 \\
Trade-off & 70.49 & 61.98 & 8.52 \\
\hline
\end{tabular}

level provides the set-points for the lower-level control system. The lower level optimizes the pressure profile to minimize losses due leakage and to provide sufficient water pressure, e.g. for high-rise buildings.

\section{Simplifying assumptions}

This paper considers an aggregate version of the Barcelona DWN, which is a representative version of the entire network developed cooperatively by the AGBAR Company and the SAC research group. In the aggregate model, some consumer demand sectors of the network are concentrated in a single point. Similarly, some tanks are aggregated in a single element and the respective actuators are considered as a single pumping station or valve. Pumping stations flows are treated as continuous variables. This means that a pumping station is modelled as being able to produce any flow in a certain range, so that an additional scheduling procedure (not addressed here) is required for individual pump operation to produce the desired flow. The aggregate network, shown in Figure 2, is comprised of 17 tanks (state variables of the dynamical network model), 61 actuators (26 pumping stations and 35 valves), 11 nodes and 25 main sectors of water demand (model disturbances). The model has been simulated and compared against real behaviour assessing its validity. The detailed information about physical parameters and other system values is reported in (Caini et al. 2009).

\section{SIMULATIONS AND RESULTS}

\section{Simulator of Barcelona DWN}

A toolbox for simulation of DWN has been developed in MATLAB/SIMULINK, which allows implementing and testing control solutions. The results presented in this section were tested against a simulator developed using this DWN simulation toolbox. Figure 3 presents the SIMULINK block diagram of the simulator. This simulator not only allows the user to test the MPC controller developed in closed loop but also to evaluate the real economic cost of the DWN operation given by the control actions derived by using such controller. With this simulator, it is possible to compare the economic cost of the manual operation of the network against the optimized one considering the MPC controller.

\section{Tuning set-up of the MPC controller}

The MPC controller developed for the optimal control of the Barcelona network uses a prediction horizon of 24 hours. The control horizon was also set to 24 hours. It is left as further research to investigate whether a smaller control horizon might be used in order to save computation time. The MPC controller is implemented using GAMS-CONPT (GAMS 2004), which is interfaced with the MATLAB environment. The demand forecast obtained from the procedure previously described in this paper, is implemented in MATLAB as separate module. The safety volumes of tanks have been determined increasing by a $20 \%$ the minimum volumes required to satisfy the demand one hour ahead of the prediction horizon. The MPC performance function, as discussed in the MPC design section, includes three terms: economic cost, safety and

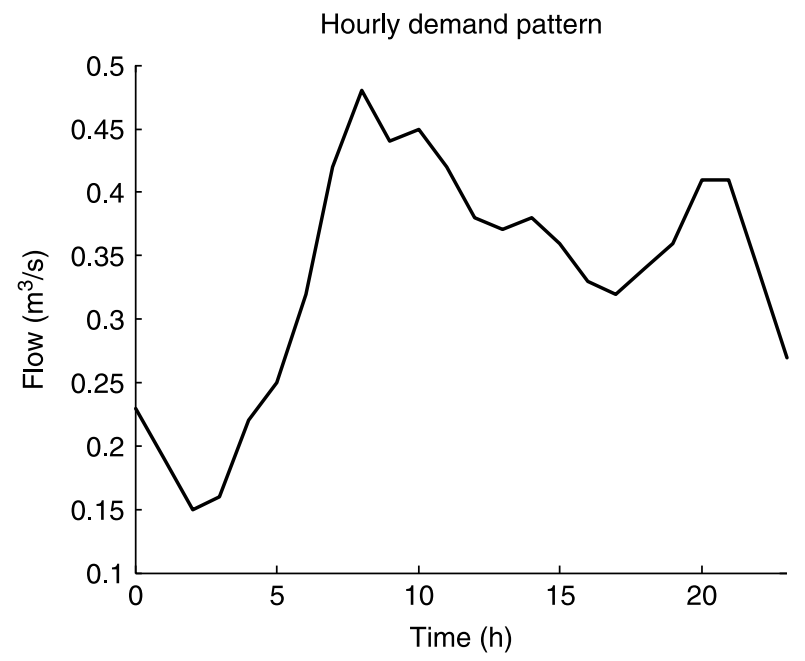

Figure 4 | Hourly pattern of a demand sector. 

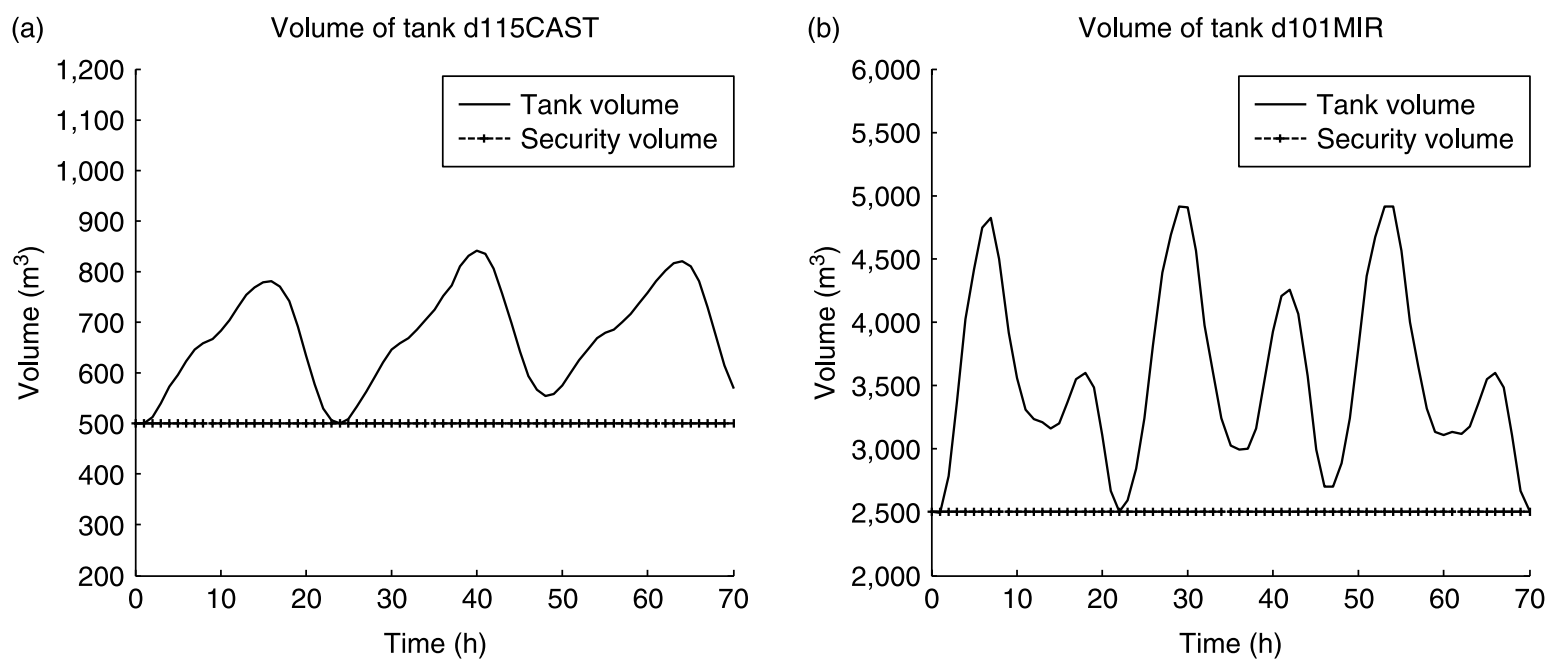

Figure $\mathbf{5}$ | Volume evolution of two selected tanks.

stability terms. The main priority is to minimize the economic cost. At the same time, safety and stability objectives must also be optimized. The relative importance of these objectives is given by the weight in the performance function. Table 1 presents the results of the tuning procedure where just one weight is emphasised at time. Numbers are not given in real economical units due to confidentiality reasons. The closed-loop results presented in the next section have been obtained by using a tuning procedure that analyses the trade off between the control objectives taking the given prioritization into account. The controller also takes advantage of the electrical cost tables so that the optimal strategy can be found trying to pump

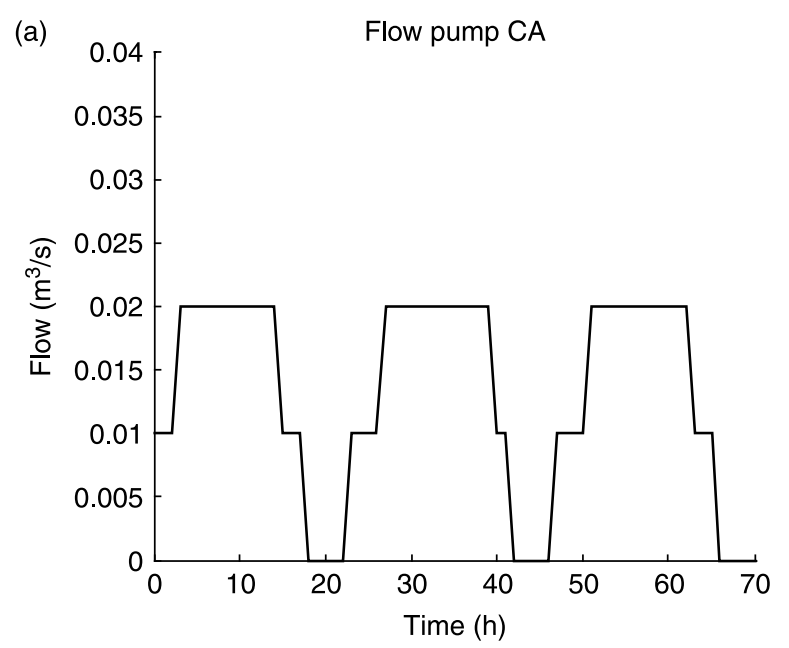

when electricity is less expensive (in Barcelona mainly during the night time).

\section{Closed-loop results}

The case study was parameterized using real data (e.g. demands, operational ranges of elements, etc.). Figure 4 shows a demand profile corresponding to the 24-hour demand distribution in one demand sector. Similar demand patterns are used on all demand locations. The test considers a periods of three consecutive days.

Figure $5 \mathrm{a}$ and $\mathrm{b}$ plot the evolution of two tanks of the Barcelona DWN during 72 hours. This figure shows that

Figure 6 | Pumped flow to the tank d115CAST and electrical cost tariff for this pump.

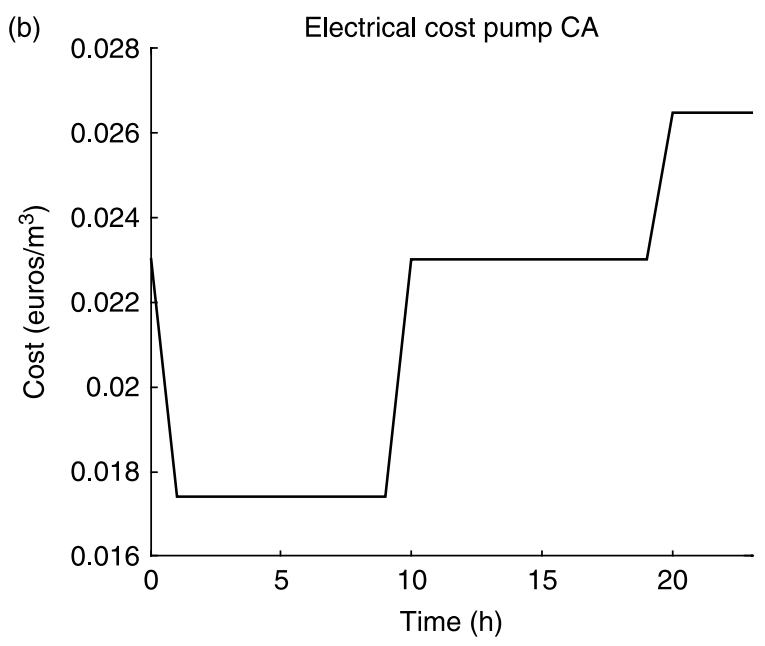




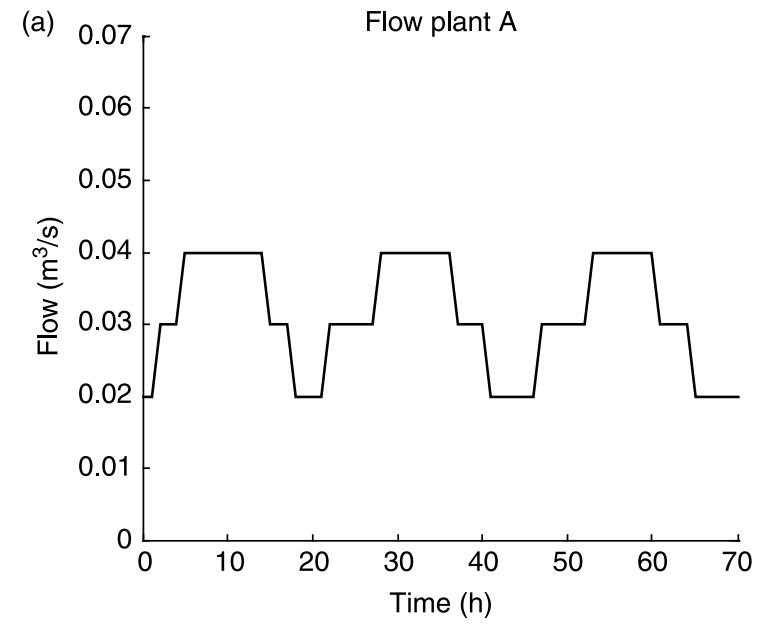

Figure 7 | Outflow from water treatment plants.

the demand may be efficiently satisfied while the safety volume is maintained at all times. This fact implies the proper working of the MPC controller, improving the performance index associated to the safety storage. Figure 6a shows the flow pumped to the tank d115CAST, whose volume evolution is presented in Figure $5 \mathrm{~b}$. It can be noticed that MPC controller decides to avoid pumping at the peak-tariff times (see Figure 6b). In Figure 5b, the volume evolution of tank d101MIR shows that, in order to supply water for the later part of the day, it is necessary to receive water during the day in addition to filling the tank at night time with lower electricity tariffs (see Figure 6b). It is important to note that the designed management strategy allows for emptying the tank close to the safety limit, so as to avoid pumping during peak-tariff time. In Figure $7 \mathrm{a}$ and $\mathrm{b}$, the total flow required from two treatment plants is presented. These figures show how the stability term in the cost function has efficiently handled the storage capability of the network to satisfy the demands with stable flows from the plants, i.e. avoiding abrupt variations of their outflows.

\section{CONCLUSIONS AND FURTHER WORK}

This paper has presented preliminary results of applying MPC techniques for flow management in a large-scale drinking water network including a telemetry/telecontrol system. The obtained results have shown the smart capabilities of MPC to generate control strategies that fill the tanks to appropriate volumes in order to meet demand, reducing water transport and production costs by taking into account time-varying electrical tariffs and by using less expensive sources while maintaining the safety tank volumes for avoiding risk of shortage in the water supply. Moreover, the use of a stability term in the performance function to be optimized by the MPC strategy provides smooth flows, especially in treatment plants outflows. This fact implies an efficient handling of the plants, avoiding performance problems of these elements. The next step of this study, currently underway, is to compare the optimal actions computed by the MPC strategy with the current strategies applied by AGBAR to the real system in order to examine their respective costs and degree of completion of the safety and stability goals.

\section{ACKNOWLEDGEMENTS}

This work has been supported by the CICYT Ref. DPI200611944 of the Spanish Science and Technology Ministry, the Juan de la Cierva Research Programme (Ref. JCI-20082438), and the DGR of Generalitat de Catalunya (SAC group Ref. 2009/SGR/1491). The authors specially thank the permanent and invaluable collaboration of AGBAR staff and the work of E. Caini in the simulation results presented in this paper. 


\section{REFERENCES}

Brdys, M. A. \& Ulanicki, B. 1994 Operational Control of Water Systems: Structures, Algorithms and Applications. Prentice Hall International.

Brdys, M. A., Grochowski, M., Gminski, T., Konarczak, K. \& Drewa, M. 2008 Hierarchical predictive control of integrated wastewater treatment systems. Control Eng. Pract. 16(6), $751-767$.

Butler, D. \& Memon, F. A. 2006 Water Demand Management. IWA Publishing.

Caini, E., Puig, V., \& Cembrano, G. 2009 Development of a Simulation Environment for Water Drinking Networks: Application to the Validation of a Centralized MPC Controller for the Barcelona Case Study. Technical Report ref. IRI-TR03-09. IRI-CSIC-UPC, Barcelona.

Camacho, E. F. \& Bordons, C. 2004 Model Predictive Control. Springer, Great Britain.

Cembrano, G., Wells, G., Quevedo, J., Pérez, R. \& Argelaguet, R. 2000 Optimal control of a water distribution network in a supervisory control system. Control Eng. Pract. 8, $1177-1188$.

GAMS 2004 The Solver Manuals. Development Corporation. Washington, DC. http://www.gams.com/

Maciejowski, J. M. 2002 Predictive Control: With Constraints. Prentice Hall.
Maksimovic, C., Butler, D. \& Memon, F. A. 2003 Advances in Water Supply Management: Proceedings of the International Conference on Computing and Control for the Water Industry. Taylor \& Francis.

Marinaki, M. \& Papageorgiou, M. 2005 Optimal Real-time Control of Sewer Networks. Springer.

Nitivattananon, V., Sadowski, E. C. \& Quimpo, R. G. 1996 Optimization of water-supply system operation. J. Water Resour. Plan. Manage. 122(5), 374-384.

Ocampo-Martinez, C. 2007 Model Predictive Control of Complex Systems including Fault Tolerance Capabilities: Application to Sewer Networks. PhD Thesis, Automatic Control Department (ESAII), Technical University of Catalonia.

Ocampo-Martínez, C., Ingimundarson, A., Puig, V. \& Quevedo, J. 2008 Objective prioritization using lexicographic minimizers for MPC of sewer networks. IEEE Trans. Control Syst. Technol. 16(1), 113-121.

Ragot, J. \& Maquin, D. 2006 Fault measurement detection in an urban water supply network. J. Process Control 16, 887-902.

Tu, M. Y., Tsai, F. \& Yeh, W. 2005 Optimization of water distribution and water quality by hybrid genetic algorithm. J. Water Resour. Plann. Manage. 131(6), 431-440.

Westphal, K. S., Vogel, R. M., Kirshen, P. \& Chapra, S. C. 2003 Decision support system for adaptive water supply management. J. Water Resour. Plann. Manage. 129(3), $165-177$. 